\title{
Three-step one-way model in terahertz biomedical detection
}

\author{
Yan Peng ${ }^{1+} \mathbb{D}^{\text {, Jeli Huang }}{ }^{2 \dagger}$, Jie Luo ${ }^{1}$, Zhangfan Yang ${ }^{1}$, Liping Wang ${ }^{1}, \mathrm{Xu} \mathrm{Wu}^{1}$, Xiaofei Zang ${ }^{1}$, Chen $\mathrm{Yu}^{2 *}$, Min Gu${ }^{3}$, \\ Qing $\mathrm{Hu}^{4}$, Xicheng Zhang ${ }^{5}$, Yiming Zhu ${ }^{1 *}$ and Songlin Zhuang ${ }^{1}$
}

\author{
* Correspondence: yuchen@tongji. \\ edu.cn; ymzhu@usst.edu.cn \\ Yan Peng and Jieli Huang the first \\ co author. \\ †Yan Peng and Jieli Huang \\ contributed equally to this work. \\ ${ }^{2}$ Department of Nephrology, Tongji \\ Hospital, Tongji University School of \\ Medicine, Shanghai 200065, China \\ 'Shanghai Key Lab of Modern \\ Optical System, Terahertz \\ Technology Innovation Research \\ Institute, University of Shanghai for \\ Science and Technology, 516 \\ Jungong Road, Shanghai 200093, \\ China \\ Full list of author information is \\ available at the end of the article
}

\begin{abstract}
Terahertz technology has broad application prospects in biomedical detection. However, the mixed characteristics of actual samples make the terahertz spectrum complex and difficult to distinguish, and there is no practical terahertz detection method for clinical medicine. Here, we propose a three-step one-way terahertz model, presenting a detailed flow analysis of terahertz technology in the biomedical detection of renal fibrosis as an example: 1) biomarker determination: screening disease biomarkers and establishing the terahertz spectrum and concentration gradient; 2) mixture interference removal: clearing the interfering signals in the mixture for the biomarker in the animal model and evaluating and retaining the effective characteristic peaks; and 3) individual difference removal: excluding individual interference differences and confirming the final effective terahertz parameters in the human sample. The root mean square error of our model is three orders of magnitude lower than that of the gold standard, with profound implications for the rapid, accurate and early detection of diseases.
\end{abstract}

Keywords: Terahertz spectroscopy, Renal fibrosis, Biomedical detection, Biomarker, Mixture interference, Individual difference

\section{Introduction}

The early detection, early diagnosis and early treatment of diseases directly affect the quality of life and survival rate of patients. Pathology diagnosis is the gold standard for the diagnosis of many diseases. The corresponding technologies include light microscopic morphological detection, immunohistochemical enzyme labelling, fluorescence in situ hybridization and gene rearrangement detection [1-5]. Light microscopic morphological detection can judge the nature of the disease by observing the morphological changes in cells but is subjective because this approach is influenced by the observer's experience and intuition. Immunochemical enzyme labelling and fluorescence in situ hybridization (FISH) both use the fluorescent labelling of specific proteins or nucleic acids for localization and quantitative labelling, and gene rearrangement detection technology is used to detect and diagnose diseases by gene sequencing of lesion tissue. However, the sample processing of these techniques is cumbersome and timeconsuming and requires large amounts of reagents and dyes, so the samples cannot be

(c) The Author(s). 2021 Open Access This article is licensed under a Creative Commons Attribution 4.0 International License, which permits use, sharing, adaptation, distribution and reproduction in any medium or format, as long as you give appropriate credit to the original author(s) and the source, provide a link to the Creative Commons licence, and indicate if changes were made. The images or other third party material in this article are included in the article's Creative Commons licence, unless indicated otherwise in a credit line to the material. If material is not included in the article's Creative Commons licence and your intended use is not permitted by statutory regulation or exceeds the permitted use, you will need to obtain permission directly from the copyright holder. To view a copy of this licence, visit http://creativecommons.org/licenses/by/4.0/. 
reused, and the judgement of results also has subjective interference. Other spectroscopic methods, such as fluorescence spectroscopy and Raman spectroscopy, can also be used for sample detection. Fluorescence spectrometry mainly uses certain fluorescent dyes to form complexes with substances that do not ordinarily emit fluorescence but are induced to emit fluorescence before determination [6-9]. The procedure is complicated, the fluorescence intensity is affected by the wavelength and temperature of the stimulated luminescence, and the data stability is poor. Raman spectroscopy exposes the sample to a strong laser and collects the scattering signal for material identification $[10,11]$. However, the high-power laser easily damages the samples, which is not conducive to secondary detection, and the detection error at low concentrations is large, so the method cannot be applied to the early quantitative analysis of diseases. Therefore, there is an urgent need for rapid, label-free, simply operated, low-sampleloss, and low-cost detection technology for the early diagnosis of diseases.

Terahertz $(\mathrm{THz})$ waves $(0.1-10 \mathrm{THz})$ lie between the millimetre wave and infrared band, which is of significant importance to the biological sciences due to providing information complementary to traditional spectroscopic measurements of low-frequency bond vibrations, hydrogen bond stretching, and bond torsions in liquids and gases [12]. Therefore, the collective behaviour (vibration and rotation) characteristics of biomolecules make THz spectroscopy a promising sensing modality for clinical diagnosis [13]. Because of these properties and its non-destructiveness, accuracy, rapidity and good penetrability [14], THz spectroscopy also has many other potential applications in several research fields, including physics [15, 16], biology [17-21], chemistry [22, 23], and medicine [24, 25]. At present, substance identification with $\mathrm{THz}$ technology is mainly based on the fingerprint peaks of compounds. Some studies have indicated that the approach can be combined with chips, algorithms, reagents and other auxiliary means to improve the recognition accuracy [18, 26-30]. However, THz technology has not been effectively applied in actual medical diagnosis or evaluation, because human tissue is a mixture of many substances, and the $\mathrm{THz}$ fingerprint spectra of these substances overlap with each other, which interferes with the quantitative analysis of disease markers or key substances. More importantly, there are individual differences between human bodies, and the proportions of various components are continually changing, which leads to a rapid decrease in the effectiveness of auxiliary means such as algorithms. To date, the application of $\mathrm{THz}$ technology to biomedical sample detection is still in the process of exploration, and there is no standardized process or model to assist the accurate diagnosis of diseases.

In this paper, we propose a three-step one-way $\mathrm{THz}$ model to analyse the application steps and processes of using $\mathrm{THz}$ spectroscopy to detect diseases. We hope that we can apply this model to improve the application of $\mathrm{THz}$ technology in research on related diseases and to realize the rapid and effective detection of pathological specimens of various diseases by using $\mathrm{THz}$ technology.

The THz three-step one-way model is divided into three steps, namely, biomarker determination, mixture interference removal, and individual difference removal. In the first step, we need to screen and determine the biomarkers or key substances (of which there can be multiple), test the THz fingerprint spectrum, and establish the gradient relationship between the biomarker concentration and $\mathrm{THz}$ parameters to lay the foundation for subsequent calibration of the disease pathological stage. Second, to verify the 
possibility of applying $\mathrm{THz}$ technology to recognize biomarkers in biological tissues (mixtures), we initially used animal models to predict and quantify these markers in accordance with scientific research ethics. It is possible to analyse the biomarkers can be identified by observing the rat pathological samples. The third step is mainly to determine whether the individual differences in the human body will affect the qualitative identification and quantitative analysis of the biomarkers by $\mathrm{THz}$ spectroscopy and to verify the validity and accuracy of the biomarker parameters. The above three steps can be implemented only in one direction; otherwise, it is easy to reach a misdiagnosis or incorrect stage identification due to individual differences or mixture identification errors.

To assess the feasibility of the $\mathrm{THz}$ three-step one-way model, we used renal fibrosis, a common chronic disease, as an example. Renal fibrosis is a common pathological manifestation of end-stage renal disease; its main pathological features are glomerulosclerosis, tubular atrophy and increased extracellular matrix deposition [31]. Studies have shown that the assessment of renal fibrosis can guide the treatment and prognosis of autologous kidneys [32] and transplanted kidneys [33]. There are three methods for the diagnosis of fibrosis: histological evaluation, imaging evaluation and biomarker detection. 1) Histology is the gold standard for the diagnosis of fibrosis and can directly observe the degree of fibrosis, but its identification depends on the subjective judgement of pathologists, and there are individual differences. In addition, in some cases, there are sampling errors in the tissues obtained from core biopsy [34]. Moreover, there are time limitations in histopathological detection: sample preparation takes a long time (approximately $15 \mathrm{~h}$ ), preventing rapid determination of the degree of fibrosis. 2) Imaging evaluation methods, including ultrasound and functional magnetic resonance imaging (MRI), are new noninvasive techniques. Renal fibrosis is reflected by observing the renal elasticity, oxygen content and blood perfusion. However, the identification of renal fibrosis is easily affected by many factors, such as blood pressure, body weight, respiratory movement and differences in subjective judgement among observers, resulting in low detection accuracy [35]. 3) The use of biomarkers in urineas a noninvasive detection method is expected to be usable to monitor the progression of renal fibrosis [36]. However, some promising biomarkers, such as microRNAs [37], still suffer from instability or lack of regularity as the disease progress. Therefore, we expect to solve these problems through the $\mathrm{THz}$ three-step one-way model.

\section{Materials and methods}

Terahertz time-domain spectroscopy (THz-TDS)

The experimental setup used here is $\mathrm{THz}$ time-domain spectroscopy. In our experiments, we used an $800 \mathrm{~nm}$ femtosecond laser with a pulse duration of $100 \mathrm{fs}$, a repetition rate of $76 \mathrm{MHz}$, and an average power of $150 \mathrm{~mW}$. The emitted laser beam was split into a pump beam and probe beam by a $50 / 50$ beam splitter. A pump beam modulated by an optical chopper was focused on a gallium arsenide (GaAs) photoconductive emitter of $\mathrm{THz}$ waves. The diverging $\mathrm{THz}$ beam was collected and focused by four off-axis paraboloid mirrors to pass though samples, and then, the probe beam was used to detect the $\mathrm{THz}$ wave by photoconductive antennas. For our experimental system, the effective bandwidth for the measured signals ranged from 0.2 to $2.0 \mathrm{THz}$, the 
spectrum resolution was $\sim 15 \mathrm{GHz}$, and the signal-to-noise ratio (SNR) was greater than 1000:1. All the spectra were averaged 256 times to ensure a high SNR. The THz pulse waveform acquisition speed is $10 \mathrm{scans} / \mathrm{s}$, so the time required to collect each set of 256 spectra is about $25.6 \mathrm{~s}$. The sample absorbance $\alpha(\omega)$ was calculated by using the following equation: $\alpha(\omega)=\log \left(\mathrm{I}_{\text {ref }}(\omega)_{/ \mathrm{Isam}}(\omega)\right) / \mathrm{d}$, where $\mathrm{d}=0.1 \mathrm{~mm}$ is the thickness of the sample, $I_{\text {sam }}(\omega)=E_{\text {sam }}(\omega) \times E_{\text {sam }}(\omega)^{\prime \prime}$ is the power spectrum of the sample, and $\mathrm{I}_{\text {ref }}(\omega)=\mathrm{E}_{\mathrm{ref}}(\omega) \times \mathrm{E}_{\mathrm{ref}}(\omega)^{*}$ is the power spectrum of the reference signal.

\section{Preparation of pure L-hydroxyproline samples}

Pure L-hydroxyproline was purchased in powder form (Sigma-Aldrich) at the highest purity available $(\geq 99 \%)$. The tablets were prepared with $30 \mathrm{mg}$ of L-hydroxyproline and $120 \mathrm{mg}$ of high-density polyethylene (powders, $40-48 \mu \mathrm{m}$ particle size), which were then pressed into discs under a pressure of 4 tons. The thickness of the sample tablet was approximately $1 \mathrm{~mm}$, and the diameter was $13 \mathrm{~mm}$. The entire $\mathrm{THz}$ spectroscopy system was enclosed in a sealed box filled with dry air (humidity $<2 \%$ ) to reduce water vapour absorption.

\section{Variable-temperature liquid nitrogen cryostat}

A variable-temperature liquid nitrogen cryostat (Optistat DN2, Oxford Instruments) equipped with high-density polyethylene windows is a commercial product designed for optical spectroscopy. The cryostat temperature can continuously vary between $77 \mathrm{~K}$ and $500 \mathrm{~K}$, with an accuracy of $\pm 0.5 \mathrm{~K}$. In our experiments, the cryostat was placed at the $\mathrm{THz}$ radiation focal point between the off-axis parabolic mirrors of the THz-TDS system. The sample was placed inside in the cryostat, and $\mathrm{THz}$ radiation was passed through the sample to obtain the fingerprint spectrum.

\section{Preparation of animal model samples}

Male Sprague-Dawley (SD) rats aged 8-12 weeks with body weights of 180-200 g were provided by the Jiesijie Animal Experimental Center (Shanghai, China). All rats were nurtured and maintained according to the 'Care and Use of Laboratory Animals' guidelines published by the National Institute of Health. The Experimental Animal Ethics Committee of Shanghai Tongji Hospital (Shanghai, China, document No. 2020-DW002) approved the research protocol. The unilateral ureteral obstruction (UUO) rat model was used to study interstitial fibrosis. A total of 20 male SD rats were randomly divided into the following four groups: the sham group represented normal kidneys (sham, $n=5)$, the UUO day 3 group represented early injury $(\mathrm{d} 3, \mathrm{n}=5)$, the UUO day 7 group represented moderate fibrosis $(\mathrm{d} 7, \mathrm{n}=5)$, and the UUO day 14 group represented severe fibrosis $(\mathrm{d} 14, \mathrm{n}=5)$. After anaesthesia by using $3 \%$ intraperitoneal pentobarbital $(2 \mathrm{ml} / \mathrm{kg})$, the UUO groups received left ureteral ligation in the sham group, and the left ureter was separated, but not ligated. On days 3, 7, and 14 after surgery, the rats were sacrificed using euthanasia. Their left kidneys were removed and homogenized and then placed into sterile tubes that were labelled. All sterile tubes were kept in a $200 \mathrm{~K}$ freezer. Then, rat left kidney homogenates were carefully mounted between two flat quartz plates with a groove thickness of $480 \mu \mathrm{m}$. 


\section{Masson staining}

After the kidneys were harvested, they were sliced axially into 3-mm-thick sections, fixed in $10 \%$ buffered formalin, embedded in paraffin, and deparaffinized by submersing the slides in 4 changes of xylene for $2 \mathrm{~min}$ each, then dehydrated in 2 changes of absolute alcohol for $2 \mathrm{~min}, 1$ change of $95 \%$ alcohol for $2 \mathrm{~min}$, and 1 change of $70 \%$ alcohol for $2 \mathrm{~min}$. The slides were then mordanted by submersion in Bouin's fixative for $1 \mathrm{~h}$ at $50-60{ }^{\circ} \mathrm{C}$, washed in gently running tap water until the yellow colour completely disappeared, rinsed in distilled 3 times, stained by submersion in Weigert's iron haematoxylin for $10 \mathrm{~min}$, rinsed in warm tap water for 15 min, stained by submersion in Biebrich scarlet-acid fuchsin for $15 \mathrm{~min}$, immersed in phosphomolybdic-phosphotungstic acid for $15 \mathrm{~min}$, and immersed in Aniline blue solution for $15 \mathrm{~min}$. Next, the slides were rinsed with distilled water 3 times, immersed in $1 \%$ acetic water for 1 to $3 \mathrm{~min}$, rinsed in distilled water 3 times, dehydrated by submersion in 4 absolute alcohol changes for 2 min each, and cleared by submersion in 4 xylene changes for 2 min each. Finally, the coverslip was mounted in resinous mounting media.

\section{Preparation of human kidney samples}

Fourteen human kidney samples were obtained from Tongji Hospital. The processing method of human kidneys was the same as that of rat kidneys. The trial was conducted in accordance with the Declaration of Helsinki and good clinical practice guidelines. The ethics committees of Tongji Hospital (Shanghai, China, document No. K-W-2021-001) approved the research protocol. All patients provided written informed consent before entering the trial.

\section{Alkaline hydrolysis $(\mathrm{AH})$}

First, kidney tissue (40 mg wet weight) was sufficiently mixed with the digest $(1 \mathrm{~mL})$. After the test tube was covered by a stopper, the mixture was heated at $96^{\circ} \mathrm{C}$ for $20 \mathrm{~min}$, and the $\mathrm{pH}$ was adjusted to 6.0. Second, distilled water was added to the test tube until the total volume reached $10 \mathrm{~mL}$. Next, diluted lysate $(4 \mathrm{~mL})$ and quantum active carbon $(30 \mathrm{mg})$ were added to the test tube and mixed sufficiently. The mixture was then centrifuged at $3500 \mathrm{rpm}$ for 10 min. The supernatant $(1 \mathrm{~mL})$ was transferred into a clean tube. The dichloramine-T concertration is $1.3 \%$. the solution including $32 \mathrm{ml}$ citrate-acetate buffer $\mathrm{pH} 6.0,4 \mathrm{ml}$ distilled water, $4 \mathrm{ml}$ isoropanol, $564 \mathrm{mg}$ dichloramin-T hydrate. Dimethylamino-benzaldehyde solution is $14 \%$ Ehrlich's reagent, including $4.5 \mathrm{~g}$ p-dimethylamino- benzaldehyde, $7.8 \mathrm{ml}$ of $70 \%$ perchloric acid, and $18.6 \mathrm{ml}$ isopropanol. The remainder of the experimental process is described in Table 1.

Finally, the content of L-hydroxyproline can be calculated according to the following formula:

$$
\begin{aligned}
\text { Hydroxyprolinecontent }\left(\frac{\mathrm{mg}}{\mathrm{g}}\right)= & \frac{\mathrm{OD}_{\text {Sample }}-\mathrm{OD}_{\text {Blank }}}{\mathrm{OD}_{\text {Standard }}-\mathrm{OD}_{\text {Blank }}} \times \text { Standardcontent }(5 \mu \mathrm{g} / \mathrm{mL}) \\
& \times \frac{\text { Totalvolume of lysate }(\mathrm{mL})}{\text { Tissuewet weight }(\mathrm{g})} \times \frac{1 \mathrm{mg}}{1000 \mu \mathrm{g}}
\end{aligned}
$$


Table 1 Experimental process of alkaline hydrolysis (AH)

\begin{tabular}{llll}
\hline & Blank tube & Standard tube & Sample tube \\
\hline $\begin{array}{l}\text { Distilled water }(\mathrm{mL}) \\
5 \mu \mathrm{g} / \mathrm{mL} \text { standard working solution }(\mathrm{mL})\end{array}$ & 1.0 & 1.0 & \\
$\begin{array}{l}\text { Supernatant }(\mathrm{mL}) \\
\begin{array}{l}\text { Dichloramine- }(\mathrm{mL}) \\
\text { Mix sufficiently and let stand for } 10 \mathrm{~min} .\end{array}\end{array}$ & 0.5 & 0.5 & 0.5 \\
$\quad \begin{array}{l}\text { Perchloric acid }(\mathrm{mL}) \\
\text { Mix sufficiently and let stand for } 10 \mathrm{~min} .\end{array}$ & 0.5 & 0.5 & 0.5 \\
$\quad$ Dimethylaminobenzaldehyde $(\mathrm{mL})$ & 0.5 & 0.5 & 0.5 \\
\hline
\end{tabular}

The reagents were mixed well, and the mixture was placed in a $60^{\circ} \mathrm{C}$ water bath for $15 \mathrm{~min}$, cooled to room temperature, and centrifuged at $3500 \mathrm{rpm}$ for $10 \mathrm{~min}$. The supernatant was taken and transferred into the spectrometer (at $550 \mathrm{~nm}$ ) which is from Thermo labsystem 353 to measure the absorption (OD values) of all tubes (distilled water was used as a blank)

\section{Liquid chromatography-mass spectrometry (LC-MS)}

Kidney tissue $(10 \mathrm{mg})$ was cut into slices and then transferred into a screw vial containing $6 \mathrm{~mol} / \mathrm{L} \mathrm{HCl}(1 \mathrm{~mL})$. After the vial cap was screwed on tightly, the mixture was heated for $6 \mathrm{~h}$ at $110^{\circ} \mathrm{C}$. The hydrolysed mixture was then centrifuged at $12000 \mathrm{rpm}$ for $10 \mathrm{~min}$ at $4{ }^{\circ} \mathrm{C}$. The supernatant $(100 \mu \mathrm{L})$ was transferred into a clean Eppendorf tube containing $10 \mu \mathrm{L}$ of internal standard $(10 \mu \mathrm{g} / \mathrm{mL}$ proline-13C in water) and acetonitrile (ACN, $480 \mu \mathrm{L}$ ). After being mixed on a vortexer at $1400 \mathrm{rpm}$ for $5 \mathrm{~min}$, the mixture was centrifuged at $12000 \mathrm{rpm}$ for $5 \mathrm{~min}$ at $4{ }^{\circ} \mathrm{C}$. The supernatant $(100 \mu \mathrm{L})$ was transferred into an Ultrafree centrifugal filter. After centrifugation at $12000 \mathrm{rpm}$ for $5 \mathrm{~min}$ at $4{ }^{\circ} \mathrm{C}$, the filtrate $(50 \mu \mathrm{L})$ was transferred into a $150 \mu \mathrm{L}$ insert fixed in a $2 \mathrm{~mL}$ vial for analysis.

The prepared sample was separated on an Agilent 1260 Infinity liquid chromatography instrument equipped with a $2.1 \times 100 \mathrm{~mm}$ Venusil ASB-C18 $3.0 \mu \mathrm{m}$ column (Agela Technologies, Tianjin, China) held at $25^{\circ} \mathrm{C}$. The solvent system consisted of water/ACN/formic acid (v/v, 949/50/3, solvent A) and methanol/formic acid (v/v, 998/ 2 ; solvent B). The gradient program started at $99 \%$ solvent A for $2.5 \mathrm{~min}$, which was decreased linearly to $83 \%$ solvent A in 1.5 min, then decreased linearly to $5 \%$ solvent A in $1 \mathrm{~min}$ and maintained for $5 \mathrm{~min}$, returned linearly to $99 \%$ solvent $\mathrm{A}$ in the following $0.5 \mathrm{~min}$, and finally equilibrated for $2.0 \mathrm{~min}$ until the next analysis. The flow rate was $0.3 \mathrm{~mL} / \mathrm{min}$. The injection volume was $5 \mu \mathrm{L}$, and the samples were kept at $10^{\circ} \mathrm{C}$ in the autosampler. Analytes were monitored by positive mode electrospray ionization tandem mass spectrometry in MRM mode on an AB Sciex QTrap6500 Mass Spectrometer (AB Sciex, Framingham, MA). By direct infusion of the single-compound solution at a concentration of $1 \mu \mathrm{M}$, the declustering potential (DP), collision energy (CE), and collision cell exit potential (CXP) for each analyte and internal standard were optimized for the acquisition of the maximum intensity of the precursor and production ions, respectively. The precursor and dominant daughter ions used to set up the transition monitored in MRM mode are presented in Table 2. The dwell time was set to $40 \mathrm{~ms}$ for

Table 2 The MRM transitions and associated parameters for the analyte and internal standard

\begin{tabular}{llllll}
\hline Compounds & Precursor ion $\left(\mathbf{m ~ z}^{-\mathbf{1}}\right)$ & Daughter ion $\left(\mathrm{m} \mathrm{z}^{-\mathbf{1}}\right)$ & $\mathrm{DP}(\mathbf{V})$ & $\mathrm{CE}(\mathbf{V})$ & $\mathbf{C X P}(\mathbf{V})$ \\
\hline L-proline-1- ${ }^{13} \mathrm{C}$ & 116.9 & 40.0 & 53.0 & 21.0 & 11.0 \\
hydroxyproline & 132.0 & 67.9 & 22.0 & 26.8 & 10.1 \\
\hline
\end{tabular}


each compound. The accuracy of the analyte ranged from $90 \sim 110 \%$, with an RSD less than $10 \%$.

\section{Results}

\section{STEP 1: biomarker determination}

By analysing the biological process and characteristics of fibrosis, we know that collagen is the main component of the extracellular matrix. The accumulation of extracellular matrix in renal tissue increases with the progression of fibrosis, and the content of collagen, as its main component, also increases. However, collagen itself is a mixture of a variety of proteins and substances, so it is not suitable for use as a biomarker for $\mathrm{THz}$ technology. Regarding L-hydroxyproline, the main component of collagen [38-40], 1) its concentration is relatively fixed in mammalian collagen (approximately 14.4\%), which provides reliability for stage evaluation; 2) its molecular weight is low (131.1), and it may have clear characteristic peaks in the THz band; and 3) it is stable and difficult to react with other substances. Therefore, L-hydroxyproline has the potential to be used to identify the concentration of collagen and the developmental stage of renal fibrosis, and it was chosen as the biomarker here.

Before the experimental measurements, we needed to confirm whether the absorption peaks of the biomarker were located within the spectral range of the $\mathrm{THz}$ time-domain spectroscopy (THz-TDS) system. Ab initio calculations based on density functional theory (DFT) were performed to study the vibrational and rotational modes as well as the intermolecular interactions. The BP86 functional and $6-31++G(d, p)$ basis set were applied. We employed a dimer model for the molecule, where the signal molecule structure of L-hydroxyproline was obtained from the ChemSpider website. Our DFT-based calculations and analyses were implemented in the Gaussian09 package [41].

Figure 1a \& b present the molecular model and the DFT results of L-hydroxyproline, where three absorption peaks are observed at $0.76,1.17$, and $1.64 \mathrm{THz}$, located within the spectral window of the THz-TDS system. The corresponding origins of these absorption peaks can be identified by DFT analysis, as shown in Table 3.

After confirming that the absorption peaks were located within the $\mathrm{THz}$ spectral window, we measured the $\mathrm{THz}$ absorption spectrum of pure L-hydroxyproline. The corresponding experimental results are shown in Fig. 1c, revealing clear and distinctive absorption peaks at $0.73,1.13$, and $1.78 \mathrm{THz}$, which agree reasonably well with the DFT results. Additionally, considering that renal tissue samples provided by the hospital will be frozen and that the characteristic peaks are dependent on the test temperature, we also tested pure L-hydroxyproline at different temperatures (290, 260, $230,200,170,140,110$, and $80 \mathrm{~K}$ ) by using a variable-temperature liquid nitrogen cryostat (see Materials and methods). The corresponding results are shown in Fig. 1g. For convenience, all spectra are offset at a fixed interval. These peaks have different frequency shifts as the temperature increases: peak 3 presents the classical redshift, while peak 1 and peak 2 exhibit unusual blueshifts. For example, at $80 \mathrm{~K}$, the absorption peaks are located at $0.64,1.02$, and $1.82 \mathrm{THz}$. To show this process more clearly, we plot the frequency shift of absorption peaks as a function of temperature in Fig. 1h. The corresponding frequency values are presented in Table 4 . 

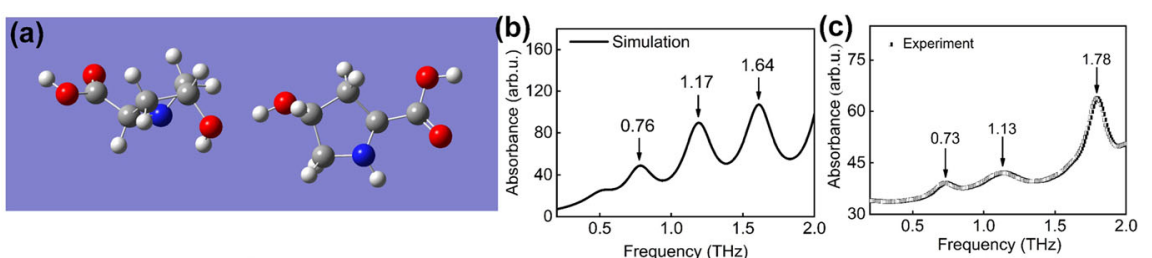

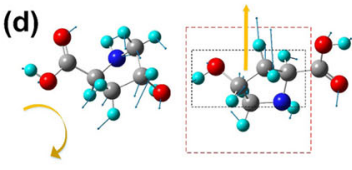

(g)

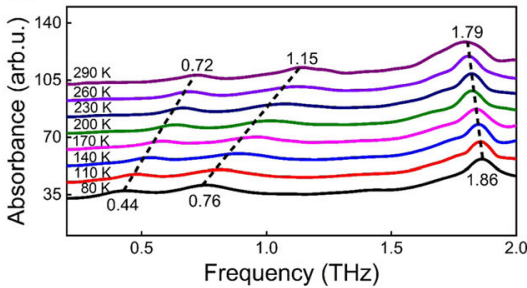

(e)

(f)

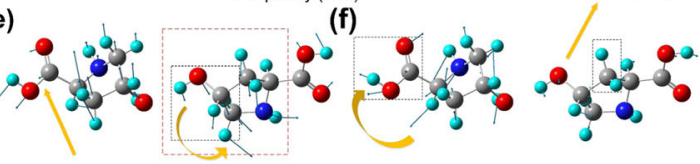

(h)

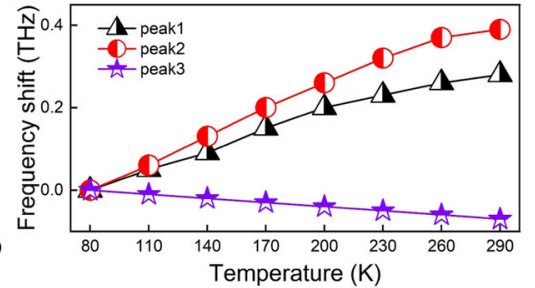

Fig. 1 Molecular simulation and THz spectrum of L-hydroxyproline. a Molecular model of L-hydroxyproline. b DFT calculated results of L-hydroxyproline. c Absorption spectrum of pure L-hydroxyproline measured experimentally by THz-TDS at room temperature. $\mathbf{d}$ to $\mathbf{f}$ Several vibration modes corresponding to the absorption peaks of L-hydroxyproline. $\mathbf{d} 0.76 \mathrm{THz}$ e $1.17 \mathrm{THz} \mathbf{f} 1.64 \mathrm{THz}$. White, grey, red, and blue balls represent $\mathrm{H}, \mathrm{C}, \mathrm{O}$, and $\mathrm{N}$ atoms, respectively. Yellow arrows indicate the vibrational direction of the atom. Black dotted and red dashed boxes refer to the dominant functional groups in the vibration modes and the entire molecular vibration of the ring, respectively. $\mathbf{g}$ Spectrum of L-hydroxyproline changes with the detection temperature from $290 \mathrm{~K}$ to $80 \mathrm{~K}$ at $30 \mathrm{~K}$ intervals. $\mathbf{h}$ Frequency shift of L-hydroxyproline absorption peaks as a function of temperature

Similar to the classical case, peak 3 undergoes a redshift as the temperature increases. This temperature dependence of the spectra can be explained by the anharmonicity of the vibrational potentials [42]. Interestingly, peak 1 and peak 2 show different behaviours, and they exhibit a blueshift as the temperature increases. Based on the DFT analysis, we deduce that the blueshift is due to competition between the intramolecular and intermolecular hydrogen bonds. As the temperature increases, thermal expansion of the intermolecular hydrogen bond partially restores the electron density of intramolecular hydrogen bonds [43], resulting in the intramolecular hydrogen bond being "harder" and its coefficient of stiffness being "stronger". Because the potential curve of the hydrogen bond near the local minimum is described by the harmonic oscillator potential, the vibration frequency is proportional to the stiffness coefficient. Therefore, as

Table 3 Calculated absorption peaks of L-hydroxyproline and vibration model analysis

\begin{tabular}{lll}
$\begin{array}{l}\text { Absorption } \\
\text { peak }\end{array}$ & $\begin{array}{l}\text { DFT } \\
\text { results }\end{array}$ & Dominant vibration model \\
\hline $\mathbf{1}$ & $\mathbf{0 . 7 6 ~ T H z}$ & $\begin{array}{l}\text { The vibration of the propyl alcohol group of the right pyrrolidine ring. Under the } \\
\text { intermolecular hydrogen bond, the left molecule vibrates back and forth following } \\
\text { the right molecule (see Fig. 1d). }\end{array}$ \\
$\mathbf{2}$ & $\mathbf{1 . 1 7 ~ T H z} \begin{array}{l}\text { The torsion and vibration of the right pyrrolidine ring. Under the intermolecular } \\
\text { hydrogen bond, the up-and-down vibration of the left L-hydroxyproline molecule } \\
\text { causes the absorption peak at 1.17 THz (see Fig. 1e). } \\
\text { The combination of the right-left vibration of the right molecule and the strong con- } \\
\text { tractions of the left molecule (see Fig. 1f). }\end{array}$ \\
\hline
\end{tabular}


Table 4 The frequency values $(\mathrm{THz})$ of pure L-hydroxyproline absorption peaks at different temperatures

\begin{tabular}{lllllllll}
\hline Temperature & $\mathbf{8 0 ~ K}$ & $\mathbf{1 1 0 ~ K}$ & $\mathbf{1 4 0 ~ K}$ & $\mathbf{1 7 0 ~ K}$ & $\mathbf{2 0 0} \mathbf{K}$ & $\mathbf{2 3 0} \mathbf{K}$ & $\mathbf{2 6 0} \mathbf{K}$ & $\mathbf{2 9 0} \mathbf{~ K}$ \\
\hline Peak 1 $(\mathrm{THz})$ & 0.44 & 0.49 & 0.53 & 0.59 & 0.64 & 0.67 & 0.70 & 0.72 \\
Peak 2 $(\mathrm{THz})$ & 0.76 & 0.82 & 0.89 & 0.96 & 1.02 & 1.08 & 1.13 & 1.15 \\
Peak 3 $(\mathrm{THz})$ & 1.86 & 1.85 & 1.84 & 1.83 & 1.82 & 1.81 & 1.80 & 1.79 \\
\hline
\end{tabular}

the temperature increases, the vibration frequency tends to increase, and the two absorption peaks undergo a blueshift.

For subsequent analysis of the relationship between the $\mathrm{THz}$ characteristic information and the evolution stage of renal fibrosis, we tested the $\mathrm{THz}$ spectrum of Lhydroxyproline at different concentrations (see Materials and methods). As shown in Fig. 2a, when the content of L-hydroxyproline was varied from $2.5 \mathrm{mg}$ to $20 \mathrm{mg}$, the corresponding $\mathrm{THz}$ absorption peaks exhibited a regular amplitude increase. For the convenience of identification, we extracted the amplitude and area of three characteristic peaks for further analysis.

As shown in Fig. 2b and c, linear fittings on the data were performed according to the Beer-Lambert law. The corresponding linear fitting function expressions are:

$$
\begin{aligned}
& \mathrm{y}_{0.73 \mathrm{THz}}=0.16257 \mathrm{x}, \mathrm{R}^{2}=0.99501 \\
& \mathrm{y}_{1.13 \mathrm{THz}}=0.17115 \mathrm{x}, \mathrm{R}^{2}=0.99566 \\
& \mathrm{y}_{1.78 \mathrm{THz}}=0.88148 \mathrm{x}, \mathrm{R}^{2}=0.99768 \\
& \mathrm{~S}_{0.73 \mathrm{THz}}=0.01755 \mathrm{x}, \mathrm{R}^{2}=0.99698 \\
& \mathrm{~S}_{1.13 \mathrm{THz}}=0.03177 \mathrm{x}, \mathrm{R}^{2}=0.99432 \\
& \mathrm{~S}_{1.78 \mathrm{THz}}=0.1566 \mathrm{x}, \mathrm{R}^{2}=0.99597
\end{aligned}
$$

$\mathrm{x}$ is the sample concentration, $\mathrm{y}$ is the amplitude $\mathrm{S}$ is the area in the expression, and $R^{2}$ is the correlation coefficient. Eq. (1)-(3) correspond to the peak amplitudes at 0.73, 1.13 and $1.78 \mathrm{THz}$, and Eq. (4)-(6) correspond to the areas of these three peaks. The corresponding $\mathrm{R}^{2}$ values show that the amplitude and area of the three peaks of $\mathrm{L}$ hydroxyproline all have a very good linear relationship with the concentration. Therefore, the concentration of L-hydroxyproline can be effectively calibrated and identified by its $\mathrm{THz}$ characteristic peak amplitude and area, which can be used to distinguish different stages of renal fibrosis.

\section{STEP 2: mixture interference removal}

After confirming that L-hydroxyproline has a clear $\mathrm{THz}$ characteristic peak that changes linearly with concentration, we started the rat test to observe whether this biomarker can be recognized in tissue samples. The unilateral ureteral obstruction (UUO) rat model was used to study interstitial fibrosis (see Materials and methods). A total of 20 male Sprague-Dawley rats were randomly divided into the following four groups: the sham group, the UUO d3 group, the UUO d7 group and the UUO d14 group. The corresponding pathologic images and $\mathrm{THz}$ spectra of the four different groups are shown in Fig. 3. 

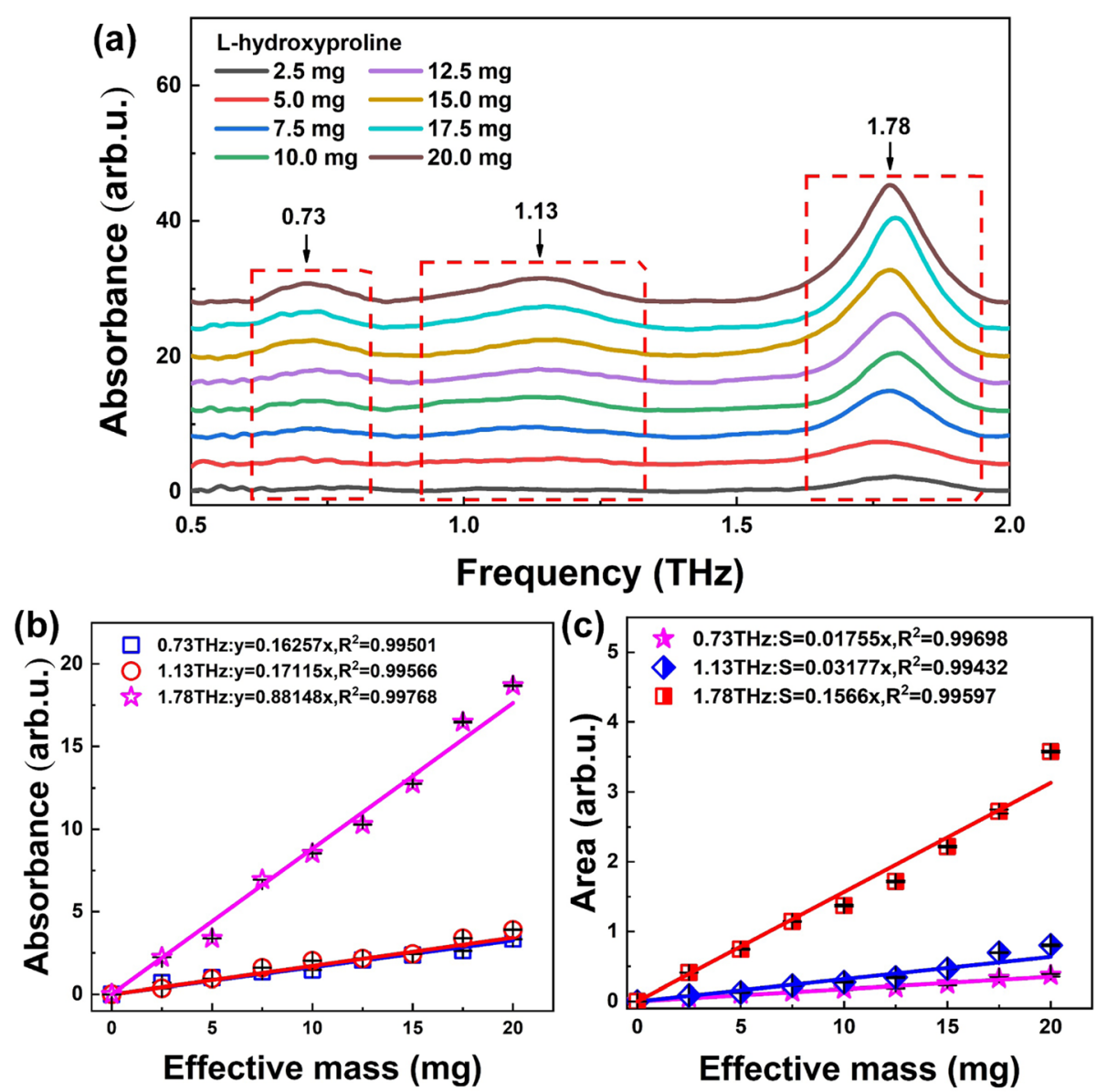

Fig. 2 Concentration gradient change of the L-hydroxyproline spectrum. a THz absorption spectra of Lhydroxyproline samples at different concentrations measured experimentally. b Absorbance at $0.73 \mathrm{THz}$ (square), $1.13 \mathrm{THz}$ (circle) and $1.78 \mathrm{THz}$ (pentagram) for different concentrations of L-hydroxyproline samples. c The area under the absorption peaks of all L-hydroxyproline samples; error bars have been labelled on each data point

Figure $3 \mathrm{a}_{1}-\mathrm{a}_{4}$ shows the pathological features of renal tissue in rats. In the sham group, the renal tissue structure was normal. Three days after the UUO operation, the renal tubules were slightly dilated, the renal interstitium was oedematous, the interstitium was widened, and the "back-to-back" characteristics were lost. There were scattered infiltrating inflammatory cells in the tubulointerstitium. Seven days after UUO, some proximal tubules showed vacuolar degeneration, epithelial cells were seen in the renal tubules, the renal tubules at the junction of the medulla and dermis medulla were obviously expanded, and a large number of inflammatory cells had infiltrated. On the 14th day after UUO, some tubules had disappeared, the collecting duct and distal renal tubule had expanded into cysts, inflammatory cell infiltration and fibroblast proliferation were obvious, and the cortex was thinner. These microscopic images are common evidence of clinical prognosis, but the accuracy of early diagnosis is low. For the early detection of fibrosis, alkaline hydrolysis $(\mathrm{AH})$ or liquid chromatography-mass spectrometry (LC-MS) could be used to quantify the hydroxyproline contents and complete the diagnosis. Although these two methods are expensive and time-consuming, there is no better alternative in clinical practice. For the convenience of comparison, we 


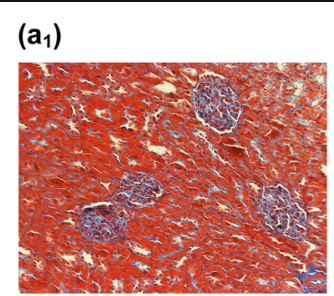

$\left(a_{2}\right)$

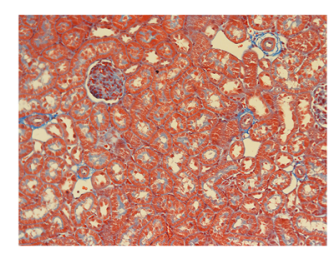

$\left(a_{3}\right)$

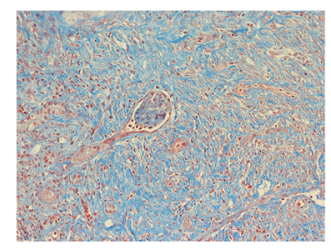

$\left(a_{4}\right)$

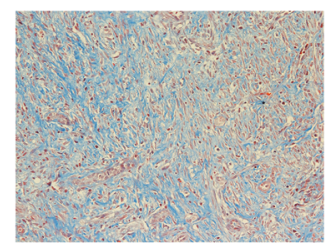

(b)

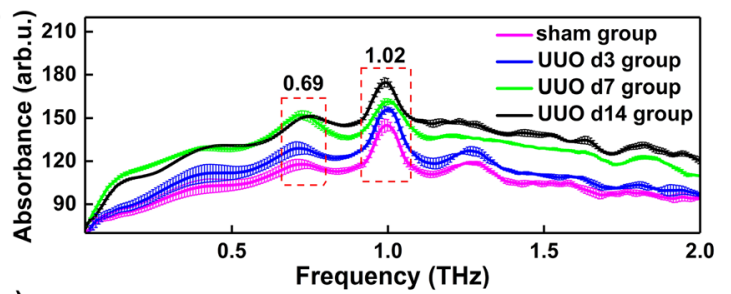

(c)

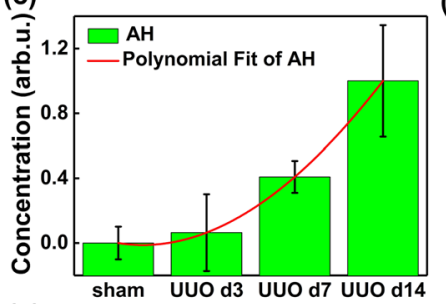

(d) $\overline{-}$ (e)

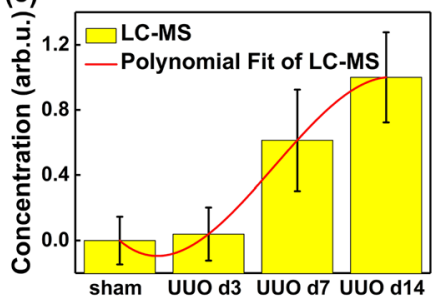

(g)

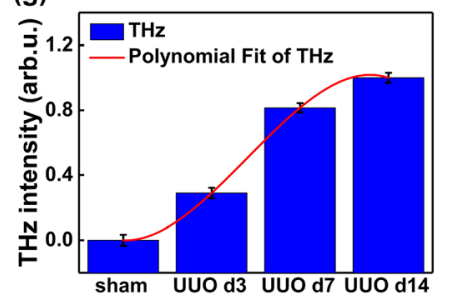

UUO Rat-AH

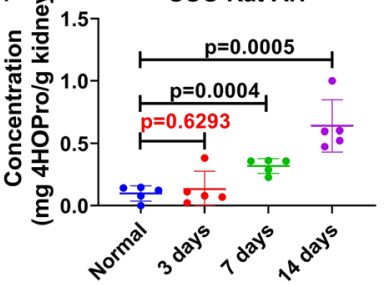

(f)

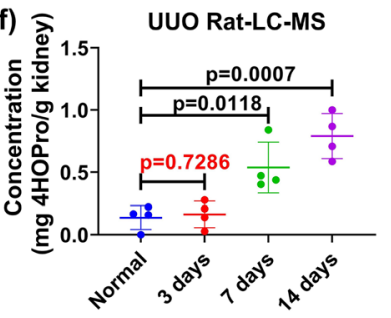

(h)

UUO Rat-THz

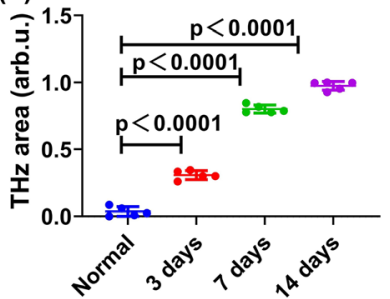

Fig. 3 Medical and THz test results in a UUO rat model. $\mathbf{a}_{\mathbf{1}}-\mathbf{a}_{\mathbf{4}}$ Kidney histologic changes in the UUO rat model. $\mathbf{a}_{1}$ Sham $\mathbf{a}_{\mathbf{2}} \cup U O \mathrm{~d} 3$ group $\mathbf{a}_{\mathbf{3}} \cup \cup O$ d7 group $\mathbf{a}_{\mathbf{4}} \cup \cup O$ d14 group (Masson staining, 200 times magnification). During each stage, five rats were tested. $\mathbf{b}$ THz absorption spectra of rat kidneys at different stages of renal fibrosis. $\mathbf{c}$ - $\mathbf{f}$ Hydroxyproline expression in different groups of $\cup \cup O$ rat models by alkaline hydrolysis (c - $\mathbf{d}$ ) and LC-MS/MS methods (e - $\mathbf{f}$. $\mathbf{g}$ - $\mathbf{h}$ THz-TDS detection in UUO kidneys by THz intensity $\mathbf{g}$ and THz area $\mathbf{h}$

prepared 5 rats for each stage of the disease. Each UUO kidney homogenate was divided into three separate parts for AH, LC-MS and THz detection.

Figure $3 \mathrm{~b}$ shows the $\mathrm{THz}$ absorption spectra of rat renal tissue at different stages after UUO operation. It can be clearly seen that the spectral shape of rat kidney tissue had changed compared with that in the spectrum of the biomarker L-hydroxyproline. In addition to the mixture causing other characteristic peaks (such as those at $0.15,0.41$, 1.27 , and $1.63 \mathrm{THz}$ ), the relative intensity and discrimination of the peaks were changed. Fortunately, the absorption peaks at 0.69 and $1.02 \mathrm{THz}$ still had high amplitude and could be effectively distinguished from the background signal. However, the characteristic peak at $1.82 \mathrm{THz}$ had undergone interference by the absorption lines of other substances in the mixture, making it difficult to distinguish effectively. Therefore, after animal model validation, only two of the three characteristic peaks were effective, and the absorption peak at $1.82 \mathrm{THz}$ was discarded. Other characteristic peaks, such as those at 0.41 and $1.27 \mathrm{THz}$, showed significant changes, but they cannot be used for 
qualitative diagnosis and quantitative analysis of diseases because their correlation with the disease cannot be determined. Based on the two characteristic peaks at 0.69 and $1.02 \mathrm{THz}$, we can see that the overall amplitude of the characteristic peaks increases regularly with the evolution of pathological stage, which indicates that the quantitative detection of L-hydroxyproline in rat kidney tissue by the THz-TDS system is expected to provide a basis for accurate medical diagnosis.

For quantitative analysis, we calculated the area under the peak of these two absorption peaks of each spectrum; that is, by integrating the spectral area of $0.59-0.84 \mathrm{THz}$ and $0.84-1.14 \mathrm{THz}$, the corresponding values were used as the judgement criteria of different fibrosis stages. The corresponding results are shown in Fig. 3c \& e \& g. Considering the different data units of the three methods, we presented the normalized values and the corresponding error bars to compare the accuracy and sensitivity in disease classification. It can be clearly seen that the measured average values of the $\mathrm{AH}$ and LC-MS methods are very close to each other. The difference between the second and first stages is very small, and the third and fourth stages are significantly different. In addition, the AH and LC-MS methods require a multi-step chemical pretreatment (see Materials and methods) and are sensitive to the operator and operation process, so the error bars are large. The THz-TDS method presents much higher values at UUO $\mathrm{d} 3$ and $\mathrm{d} 7$, and the corresponding error bars are much smaller than those of $\mathrm{AH}$ and LC-MS because THz-TDS is a physical spectral detection method that requires no preprocessing, reducing sample loss and maximizing the effective signal. In addition, the non-destructiveness and repeatability of THz-TDS measurements greatly decrease the error rate. To help clarify the standard deviation, these data are polynomially fitted as follows:

$$
\begin{aligned}
& \text { LC-MS }: y=1.22-2.08 \mathrm{x}+0.99 \mathrm{x}^{2}-0.12 \mathrm{x}^{3} \\
& \text { AH }: \mathrm{y}=0.25-0.41 \mathrm{x}+0.17 \mathrm{x}^{2}-0.01 \mathrm{x}^{3} \\
& \text { THz-TDS }: \mathrm{y}=0.53-1.13 \mathrm{x}+0.70 \mathrm{x}^{2}-0.10 \mathrm{x}^{3}
\end{aligned}
$$

where $\mathrm{x}$ represents the UUO days, and y represents the signal intensity detected by different methods. Mathematically, the $\mathrm{THz}$ fitting curve has the highest slope at low concentrations among all the methods; i.e., the $\mathrm{THz}$ method has higher sensitivity for lower-concentration samples. On the other hand, the $0.675 \%$ error bars of $\mathrm{THz}$ measurements (VS the $11.155 \%$ and $11.914 \%$ error bars of AH and LC-MS, respectively) obtained from the multiple measurements also indicate its high repeatability in identifying the presence and concentration of the biomarker L-hydroxyproline in renal tissues.

Furthermore, to quantitatively compare the differences between these three test methods of AH, LC-MS, and THz-TDS, we calculated the standard deviation and root mean square error for the four stages of the UUO rat model, as presented in Table 5 . The differences in magnitude $\left(\mathrm{S}_{\mathrm{THz}}: \mathrm{S}_{\mathrm{AH}}: \mathrm{S}_{\mathrm{LC}-\mathrm{MS}}=10^{-2}: 10^{-1}: 10^{-1}, \mathrm{RMSE}_{\mathrm{THz}}\right.$ $\mathrm{RMSE}_{\mathrm{AH}}: \mathrm{RMSE}_{\mathrm{LC}-\mathrm{MS}}=10^{-5}: 10^{-2}: 10^{-2}$ ) directly prove that the $\mathrm{THz}$ method has a higher accuracy and sensitivity than AH and LC-MS. These results are highly useful in the early identification of the biomarker L-hydroxyproline with greater confidence.

We analysed the results of the LC-MS, AH and THz tests by using the one-way analysis of variance (ANOVA) method, as shown in Fig. $3 \mathrm{~d} \& \mathrm{f} \& \mathrm{~h}$. It can be clearly seen that for both the LC-MS and AH methods, the $P$ values were all greater than 0.05 
Table 5 The standard deviation and root mean square error for the four stages of the UUO rat model

\begin{tabular}{|c|c|c|c|c|c|c|}
\hline \multirow{2}{*}{$\begin{array}{l}\text { UUO rat } \\
\text { kidney } \\
\text { samples }\end{array}$} & \multicolumn{3}{|c|}{ Standard deviation (S) } & \multicolumn{3}{|c|}{ Root mean square error (RMSE) } \\
\hline & $\mathrm{AH}$ & LC-MS & $\mathrm{THz}$ & $\mathrm{AH}$ & LC-MS & $\mathrm{THz}$ \\
\hline sham & 1.01E-01 & $1.38 \mathrm{E}-01$ & $3.38 \mathrm{E}-02$ & 1.06E-02 & $1.62 \mathrm{E}-02$ & $8.26 \mathrm{E}-05$ \\
\hline UUO d3 & $2.37 E-01$ & $1.54 \mathrm{E}-01$ & $3.20 \mathrm{E}-02$ & $5.14 \mathrm{E}-02$ & $1.86 \mathrm{E}-02$ & $6.39 \mathrm{E}-05$ \\
\hline UUO d7 & $9.79 \mathrm{E}-02$ & 2.93E-01 & 2.86E-02 & 4.97E-03 & 2.80E-02 & 3.97E-05 \\
\hline UUO d14 & 3.45E-01 & 2.60E-01 & $3.10 \mathrm{E}-02$ & 3.03E-02 & $1.44 \mathrm{E}-02$ & $4.31 \mathrm{E}-05$ \\
\hline Average & $1.95 \mathrm{E}-01$ & 2.11E-01 & $3.14 \mathrm{E}-02$ & 2.43E-02 & 1.93E-02 & 5.73E-05 \\
\hline
\end{tabular}

$\left(P_{\mathrm{AH}}=0.6293, \mathrm{P}_{\mathrm{MS}}=0.7286\right)$ in the second stage (Figs. 3d and 1f), which indicated that the early stage of fibrosis could not be effectively distinguished. By comparison, $\mathrm{THz}$ detection showed good differentiation (Fig. 3h). For the third and fourth stages, all three methods effectively identified fibrosis, but the $\mathrm{THz}$ method had a smaller error and higher discrimination. Therefore, the accuracy of early diagnosis for the $\mathrm{THz}$ method is much higher than that of the other two conventional methods.

\section{STEP 3: individual difference removal}

After confirming the feasibility of tissue detection in a rat model, we carried out an experiment with human kidney tissue. Because samples of human renal fibrosis tissue are very rare and the total amount of each sample is small, we first adopted the test mode of non-destructive detection with the $\mathrm{THz}$ method and then evaluated the degree of fibrosis by histopathology and the AH method (the amount of single tissue was not enough to support both the AH and LC-MS methods, so we chose only the conventional AH method). According to the area of renal fibrosis, the patients were divided into a normal group and a fibrosis group. The general information on the two groups is shown in Table 6.

According to Masson staining, 14 renal tissues were divided into a normal group $(n=4)$ and a fibrosis group $(n=10)$. The average age of the normal group was $50.00 \pm$ 9.41 years (mean eGFR $66.75 \pm 6.28 \mathrm{~mL} / \mathrm{min}$ ), and the fibrotic area was $4.00 \pm 0.82 \%$; the average age of the fibrosis group was $57.00 \pm 3.25$ years, the average eGFR was $65.36 \pm 8.30 \mathrm{~mL} / \mathrm{min}$, and the fibrotic area was $30.40 \pm 8.50 \%$. Figure 4a shows a pathological image of normal human kidney tissue. The glomerular capillary loop is well opened, and the renal tubules are back-to-back. No obvious tubular atrophy, interstitial fibrosis or inflammatory cell infiltration was found. Figure $4 \mathrm{~b}$ presents a pathological image of a human fibrotic kidney with obvious glomerulosclerosis, tubular atrophy with interstitial fibrosis and inflammatory cell infiltration.

The THz absorption spectra of human samples are shown in Fig. 4c. The spectra of human kidney tissue changed again compared to those of the rat model. The central frequency of the first characteristic peak at $0.69 \mathrm{THz}$ in the rat sample redshifted slightly to $0.64 \mathrm{THz}$, and the central frequency of the characteristic peak at $1.02 \mathrm{THz}$ did not change; the biomarker-unrelated peaks at $0.15,0.41$, 1.27, and $1.63 \mathrm{THz}$ in Fig. 3b no longer exhibit obvious or regular changes in Fig. 4c. We speculate that this is because the rats in the UUO model were fed uniformly and injected quantitatively. However, the individual differences among 
Table 6 Clinical characteristics of 14 patients

\begin{tabular}{lllll}
\hline Groups & $\begin{array}{l}\text { Sex } \\
(\mathbf{M : F})\end{array}$ & $\begin{array}{l}\text { Age } \\
(\mathbf{y r})\end{array}$ & $\begin{array}{l}\text { eGFR } \\
\text { (ml/min) }\end{array}$ & \multicolumn{2}{c}{$\begin{array}{l}\text { Fibrotic area } \\
\mathbf{( \% )}\end{array}$} \\
\hline Normal & $4: 0$ & $50.00 \pm 9.41$ & $66.75 \pm 12.55$ & $4.00 \pm 0.41$ \\
fibrosis & $6: 4$ & $57.00 \pm 3.25$ & $63.80 \pm 28.97$ & $30.40 \pm 8.50$ \\
\hline
\end{tabular}

(a)

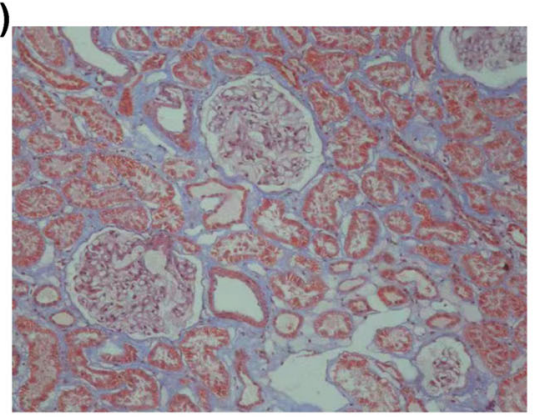

(b)

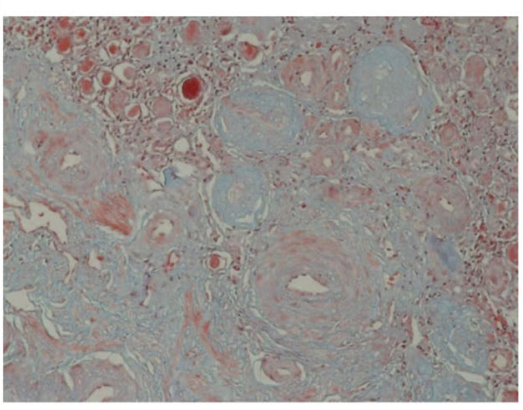

(c)

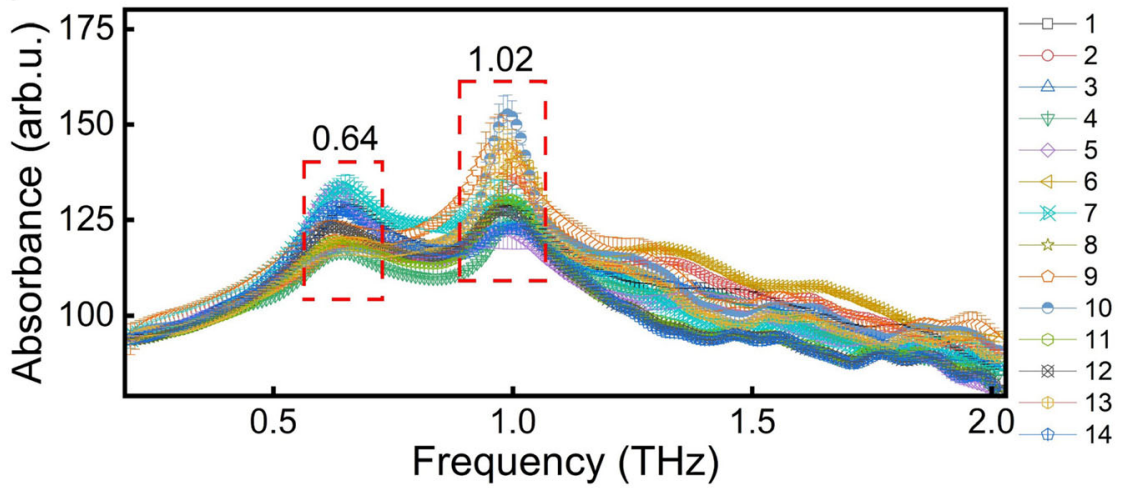

(d)

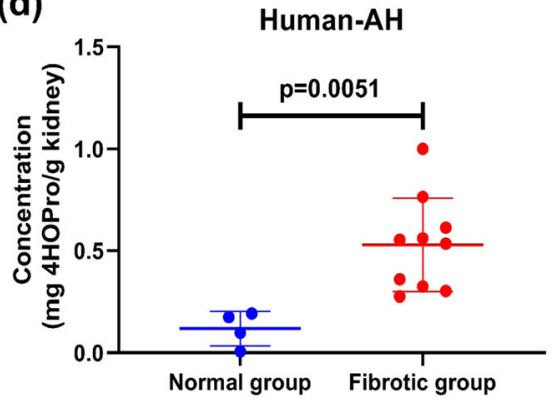

(e)
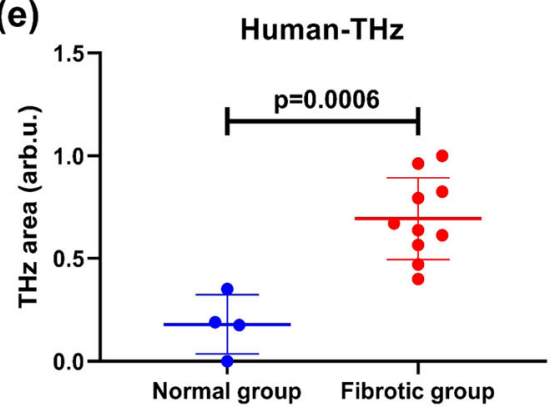

Fig. 4 Medical and THz test results of human kidney tissues. a Normal tissue image. b Fibrosis tissue image. c THz absorption spectra of 14 samples of human kidneys. Lines with different colours represent different human kidney samples, and the corresponding error bars are labelled. The red dotted box shows the $\mathrm{THz}$ spectra of the biomarker L-hydroxyproline. The numbers 1-14 represent the 14 human renal fibrosis samples. $\mathbf{d}$-e The difference between human normal and fibrotic tissues by the AH and THz-TDS methods. All data are normalized to their maximum 
human bodies (the proportion and content of each component varies with individual age, sex, physique, nutrition, exercise and other factors) will change the centre frequency of the overlapping spectrum, which causes the spectrum to no longer have obvious regular changes. However, the content of hydroxyproline, a common characteristic of renal fibrosis patients, increased significantly, making its characteristic peak still more significant and able to be effectively identified.

As in UUO, we integrated the area under the peak of the two characteristic peaks at 0.64 and $1.02 \mathrm{THz}$, corresponding to the frequency range of $0.50-0.86 \mathrm{THz}$ and $0.86-$ $1.19 \mathrm{THz}$, respectively, as shown in the red box in Fig. 4c. The relevant data and AH test results were divided into two groups, normal and pathological, as shown in Fig. 4d and e. The results showed that the content of hydroxyproline and the area under the peaks by THz-TDS in renal fibrotic and normal tissues were significantly increased, and there was a significant difference between the two groups by $\mathrm{THz}$ intensity and $\mathrm{THz}$ peak area assessment. Considering these results and the rapidity, simple operation and losslessness of the $\mathrm{THz}$ method, we conclude that the overall effect of the $\mathrm{THz}$ method is much better than that of the AH method.

\section{Discussion}

Now, we can deduce that these series of experiments directly prove the high sensitivity, accuracy and early recognition rate of $\mathrm{THz}$ methods for renal fibrosis. This sensitivity to different stages of renal fibrosis can be directly used for the dynamic monitoring of chronic kidney disease. This good performance may be due to four factors: (1) the high directionality of the THz beam yields a high collection efficiency; (2) the transmission-type detection results in a strong absorption of $\mathrm{THz}$ waves by each biomarker molecule in the sample; (3) the detection process for the $\mathrm{THz}$ method has only one step-inserting the sample into the system-which is quick and has no mass loss or denaturation; and (4) the nondestructive detection of the $\mathrm{THz}$ method allows repeated measurements so that we can obtain error bars to quantify the reliability of the result (a single $\mathrm{THz}$ measurement takes only a few seconds). Finally, the corresponding spectrum contains all the useful information. In contrast, the AH and LC-MS methods both use multiple and complex chemical reactions followed by spectral analyses. The more complex the workflows are, the more operation steps there are, which inevitably results in a reduced amount of sample and thereby a decreased signal.

So far, we have completed the three-step one-way model of the $\mathrm{THz}$ method: first, we screened the biomarkers of diseases to confirm whether the $\mathrm{THz}$ characteristic peaks exist in the effective detection range; second, by observing the interference degree of the mixture on the characteristic peaks of the biomarker by rat tissues, we confirmed the effective and recognizable characteristic peaks; and third, we excluded the influence of individual differences in human tissues and confirmed the most effective and recognizable characteristic peaks. Finally, the $\mathrm{THz}$ characteristic peak can be used effectively, allowing accurate comparison and evaluation. This one-way process gradually eliminates the interference of uncertain factors and other non-disease-related substances and establishes a standardized process for the rapid and noninvasive diagnosis of clinical diseases by the $\mathrm{THz}$ method. 


\section{Conclusion}

In summary, we provide a "THz three-step one-way" model for disease detection, which details the process of $\mathrm{THz}$ dynamic monitoring of disease evolution and the method of eliminating interference. Compared with AH and LC-MS, THzTDS can improve the accuracy, sensitivity and detection speed of diagnosis. Its high sensitivity, high accuracy and high speed highlight its potential in the early diagnosis, staging evaluation and disease monitoring of biological diseases. In the future, this kind of dynamic monitoring is expected to achieve real-time imaging during operation, which can greatly improve the accuracy of surgery.

\section{Supplementary Information}

The online version contains supplementary material available at https://doi.org/10.1186/s43074-021-00034-0.

Additional file 1: Figure S1. THz-TDS used in the experiment. Figure S2. Simplified diagram of THz-TDS.

\section{Acknowledgements}

The authors are grateful to Professor Bo Song for fruitful discussions.

\section{Authors' contributions}

$Y P, J H, Y Z$ and $C Y$ conceived the study and designed the experiments. $J L$ and $Z Y$ performed the fibrosis experiments. LW, XW and XZ designed and performed theoretical simulations. $J H$ and CY provided all samples, provided expertise for fibrosis progression, and performed the AH and LC-MS experiments in the hospital. MG, $\mathrm{QH}, \mathrm{XZ}$ and SZ provided useful advice. YP and JH wrote the manuscript. The authors read and approved the final manuscript.

\section{Funding}

This work is financially supported by NSFC $(61922059,81961138014,61805140,81873609,61771314)$ and the Terahertz Science and Technology Frontier Project (6198802).

\section{Availability of data and materials}

The calculation and experiment data that support the works of this study are available from the corresponding authors on reasonable request.

\section{Declarations}

Competing interests

The authors declare no competing interests.

\section{Author details}

${ }^{1}$ Shanghai Key Lab of Modern Optical System, Terahertz Technology Innovation Research Institute, University of Shanghai for Science and Technology, 516 Jungong Road, Shanghai 200093, China. ${ }^{2}$ Department of Nephrology, Tongji Hospital, Tongji University School of Medicine, Shanghai 200065, China. ${ }^{3}$ Laboratory of Artificial-Intelligence Nanophotonics, University of Shanghai for Science and Technology, Shanghai 200093, P. R. China. ${ }^{4}$ Department of Electrical Engineering and Computer Science and Research Laboratory of Electronics, Massachusetts Institute of Technology, Cambridge, MA 02139, USA. ${ }^{5}$ The Institute of Optics, University of Rochester, Rochester, New York 14627, USA.

Received: 19 April 2021 Accepted: 11 June 2021

Published online: 23 July 2021

\section{References}

1. Lucchinetti CF, Popescu BF, Bunyan RF, Moll NM, Roemer SF, Lassmann H, et al. Inflammatory cortical demyelination in early multiple sclerosis. N Engl J Med. 2011;365(23):2188-2197. https://doi.org/10.1056/NEJMoa1100648.

2. Longerich T, Endris V, Neumann O, Rempel E, Kirchner M, Abadi Z, et al. RSPO2 gene rearrangement: a powerful driver of $\beta$-catenin activation in liver tumours. Gut. 2019;68(7):1287-96. https://doi.org/10.1136/gutjnl-2018-317632.

3. Balázs M, Koroknai V, Szász I, Ecsedi S. Detection of CCND1 Locus Amplification by Fluorescence In Situ Hybridization. Methods Mol Biol. 2018;1726:85-100. https://doi.org/10.1007/978-1-4939-7565-5_9.

4. Worlikar T, Mendiratta-Lala M, Vlaisavljevich E, Hubbard R, Shi J, Hall TL, et al. Effects of histotripsy on local tumor progression in vivo Orthotopic rodent liver tumor model. BME Frontiers. 2020;1-14. https://doi.org/10.34133/2020/98303 04.

5. Stephens PJ, McBride DJ, Lin ML, Varela I, Pleasance ED, Simpson JT, et al. Complex landscapes of somatic rearrangement in human breast cancer genomes. Nature. 2009:462(7276):1005-10. https://doi.org/10.1038/nature08645

6. Potts PJ, Ellis AT, Kregsamer P, Streli C. X-ray fluorescence spectrometry. J Anal At Spectrom. 1999;14(11):1773-99. 
7. Höhne J, Hohenberger C, Proescholdt M, Riemenschneider MJ, Wendl C, Brawanski A, et al. Fluorescein sodium-guided resection of cerebral metastases-an update. Acta Neurochir (Wien). 2017;159(2):363-7. https://doi.org/10.1007/s00701-01 6-3054-3.

8. Stummer W, Tonn JC, Goetz C, Ullrich W, Stepp H, Bink A,et al. 5-Aminolevulinic acid-derived tumor fluorescence: the diagnostic accuracy of visible fluorescence qualities as corroborated by spectrometry and histology and postoperative imaging. Neurosurgery. 2014;74(3):310-9. discussion 319-320. https://doi.org/10.1227/NEU.0000000000000267.

9. Ishizawa T, Fukushima N, Shibahara J, Masuda K, Tamura S, Aoki T, et al. Real-time identification of liver cancers by using indocyanine green fluorescent imaging. Cancer. 2009;115(11):2491-504. https://doi.org/10.1002/cncr.24291.

10. Zhu Y, Zheng W, Wang W, et al. Raman tensor of layered black phosphorus. PhotoniX. 2020;1:17.

11. 11.You AYF, Bergholt MS, St-Pierre JP, Kit-Anan W, Pence IJ, Chester AH, et al. Raman spectroscopy imaging reveals interplay between atherosclerosis and medial calcification in the human aorta. Sci Adv. 2017;3(12):e1701156. https://doi. org/10.1126/sciadv.1701156.

12. Peng Y, Shi C, Wu X, Zhu Y, Zhuang S. Terahertz Imaging and Spectroscopy in Cancer Diagnostics: A Technical Review. BME Frontiers. 2020;2547609.

13. Peng Y, Shi C, Zhu Y, Gu M, Zhuang S. Terahertz spectroscopy in biomedical field: a review on signal-to-noise ratio improvement. PhotoniX. 2020;1(1). https://doi.org/10.1186/s43074-020-00011-z.

14. Ge H, Jiang $Y$, Lian F, Zhang $Y$, Xia S. Quantitative determination of aflatoxin B1 concentration in acetonitrile by chemometric methods using terahertz spectroscopy. Food Chem. 2016;209:286-92. https:/doi.org/10.1016/.j.foodchem.2016.04.070.

15. Wang Z, Wu J, Yang W, Bera AK, Kamenskyi D, Islam ATMN, et al. Experimental observation of Bethe strings. Nature. 2018;554(7691):219-23. https://doi.org/10.1038/nature25466.

16. Chanana A, Liu X, Zhang C, Vardeny ZV, Nahata A. Ultrafast frequency-agile terahertz devices using methylammonium lead halide perovskites. Sci Adv. 2018;4(5):eaar7353. https://doi.org/10.1126/sciadv.aar7353.

17. Lu S, Zhang X, Zhang Z, Yang Y, Xiang Y. Quantitative measurements of binary amino acids mixtures in yellow foxtail millet by terahertz time domain spectroscopy. Food Chem. 2016;211:494-501. https:/doi.org/10.1016/j.foodchem.2016.05.079.

18. Peng Y, Shi C, Xu M, Kou T, Wu X, Song B, et al. Qualitative and quantitative identification of components in mixture by terahertz spectroscopy. IEEE Trans Terahertz Sci Technol. 2018;8(6):696-701. https://doi.org/10.1109/ TTHZ.2018.2867816.

19. Wu $X$, Dai $Y$, Wang $L$, Peng $Y$, Lu L, Zhu $Y$, et al. Diagnosis of methylglyoxal in blood by using far-infrared spectroscopy and o-phenylenediamine derivation. Biomed. Opt. Express. 2020;11(2):963-70. https://doi.org/10.13 64/BOE.381542.

20. Stantchev RI, Sun B, Hornett SM, Hobson PA, Gibson GM, Padgett MJ, et al. Noninvasive, near-field terahertz imaging of hidden objects using a single-pixel detector. Sci Adv. 2016;2(6):e1600190. https://doi.org/10.1126/scia dv. 1600190 .

21. Lee SH, Lee D, Choi MH, Son JH, Seo M. Highly sensitive and selective detection of steroid hormones using terahertz molecule-specific sensors. Anal Chem. 2019;91(10):6844-9. https://doi.org/10.1021/acs.analchem.9b01066.

22. Elgabarty H, Kampfrath T, Bonthuis DJ, Balos V, Kaliannan NK, Loche P, et al. Energy transfer within the hydrogen bonding network of water following resonant terahertz excitation. Sci Adv. 2020;6(17):eaay7074. https://doi.org/10.1126/sciadv.aay7074.

23. El Haddad J, de Miollis F, Bou Sleiman J, Canioni L, Mounaix P, Bousquet B. Chemometrics applied to quantitative analysis of ternary mixtures by terahertz spectroscopy. Anal Chem. 2014;86(10):4927-33. https://doi.org/10.1021/ac500253b.

24. Wu X, Wang L, Peng Y, Wu F, Cao J, Chen X, et al. Quantitative analysis of direct oral anticoagulant rivaroxaban by terahertz spectroscopy. Analyst. 2020;145(11):3909-15. https://doi.org/10.1039/D0AN00268B.

25. Du SQ, Li H, Xie L, Chen L, Peng Y, Zhu YM, et al. Vibrational frequencies of anti-diabetic drug studied by terahertz timedomain spectroscopy. Appl Phys Lett. 2012;100:143702.

26. Sommer S, Koch M, Adams A. Terahertz time-domain spectroscopy of plasticized poly(vinyl chloride). Anal Chem. 2018; 90(4):2409-13. https://doi.org/10.1021/acs.analchem.7b04548.

27. Gu H, Shi C, Wu X, Peng Y. Molecular methylation detection based on terahertz metamaterial technology. Analyst. 2020;145(20):6705-12. https://doi.org/10.1039/D0AN01062F.

28. Peng $Y$, Yuan $X$, Zou $X$, Chen $W$, Huang H, Zhao H, et al. Terahertz identification and quantification of neurotransmitter and neurotrophy mixture. Biomed Opt Express. 2016;7(11):4472-9. https://doi.org/10.1364/BOE.7.004472.

29. Li T, Ma H, Peng Y, Chen X, Zhu Z, Wu X, et al. Gaussian numerical analysis and terahertz spectroscopic measurement of homocysteine. Biomed Opt Express. 2018;9(11):5467-76. https://doi.org/10.1364/BOE.9.005467.

30. Ajito K, Ueno Y, Kim JY, Sumikama T. Capturing the freeze-drying dynamics of NaCl nanoparticles using $\mathrm{THz}$ spectroscopy. J Am Chem Soc. 2018;140(42):13793-7. https://doi.org/10.1021/jacs.8b07828.

31. Zhou D, Liu Y. Renal fibrosis in 2015: Understanding the mechanisms of kidney fibrosis. Nat Rev Nephrol. 2016;12(2):6870. https://doi.org/10.1038/nrneph.2015.215

32. Malvar A, Pirruccio P, Alberton V, Lococo B, Recalde C, Fazini B, et al. Histologic versus clinical remission in proliferative lupus nephritis. Nephrol Dial Transplant. 2017;32(8):1338-44. https://doi.org/10.1093/ndt/gfv296.

33. Mengel M, Chapman JR, Cosio FG, Cavaille-Coll MW, Haller H, Halloran PF, et al. Protocol biopsies in renal transplantation: insights into patient management and pathogenesis. Am J Transplant. 2007;7(3):512-7. https://doi.org/1 0.1111/j.1600-6143.2006.01677.x.

34. Zhuo L, Wang H, Chen D, Lu H, Zou G, Li W. Alternative renal biopsies: past and present. Int Urol Nephrol. 2018;50(3): 475-9. https://doi.org/10.1007/s11255-017-1668-x.

35. Torres HR, Queirós S, Morais P, Oliveira B, Fonseca JC, Vilaça JL. Kidney segmentation in ultrasound, magnetic resonance and computed tomography images: a systematic review. Comput Meth Prog Bio. 2018;157:49-67. https://doi.org/10.101 6/j.cmpb.2018.01.014.

36. Zhang $X Q$, Li X, Zhou WQ, Liu X, Huang JL, Zhang YY, et al. Serum Lysyl oxidase is a potential diagnostic biomarker for kidney fibrosis. Am J Nephrol. 2020;51(11):907-18. https://doi.org/10.1159/000509381.

37. Nakhjavani M, Etemadi J, Pourlak T, Mirhosaini Z, Zununi Vahed S, Abediazar S. Plasma levels of miR-21, miR-150, miR423 in patients with lupus nephritis. Iran J Kidney Dis. 2019;13(3):198-206.

38. Hewitson TD, Smith ER, Samuel CS. Qualitative and quantitative analysis of fibrosis in the kidney. Nephrology (Carlton). 2014;19(11):721-6. https://doi.org/10.1111/nep.12321. 
39. Samuel CS. Determination of collagen content, concentration, and sub-types in kidney tissue. Methods Mol Biol. 2009; 466:223-35. https://doi.org/10.1007/978-1-59745-352-3_16.

40. Skibba M, Hye Khan MA, Kolb LL, Yeboah MM, Falck JR, Amaradhi R, et al. Epoxyeicosatrienoic acid analog decreases renal fibrosis by reducing epithelial-to-mesenchymal transition. Front Pharmacol. 2017;8:406. https:/doi.org/10.3389/fphar.2017.00406.

41. Frisch MJ, Trucks G, Schlegel HB, Scuseria GE, Robb MA, Cheeseman J, et al. Gaussian 09 Revision A.1: Gaussian Inc; 2009.

42. Joseph J, Jemmis ED. Red-, blue-, or no-shift in hydrogen bonds: a unified explanation. J Am Chem Soc. 2007;129(15): 4620-32. https://doi.org/10.1021/ja067545z.

43. Shen YC, Upadhya PC, Linfield EH, Davies AG. Temperature-dependent low-frequency vibrational spectra of purine and adenine. Appl Phys Lett. 2003;82(14):2350-2. https://doi.org/10.1063/1.1565680.

\section{Publisher's Note}

Springer Nature remains neutral with regard to jurisdictional claims in published maps and institutional affiliations.

Submit your manuscript to a SpringerOpen ${ }^{\odot}$ journal and benefit from:

- Convenient online submission

Rigorous peer review

- Open access: articles freely available online

- High visibility within the field

- Retaining the copyright to your article 